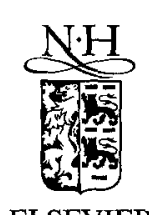

Surface Science 331-333 (1995) 1110-1114

surface science

\title{
Experimental investigation of criteria for thermal roughening
}

\author{
Petra E. Hegeman * , Harold J.W. Zandvliet, Gerard A.M. Kip, Bart A.G. Kersten, \\ Bene Poelsema
}

Faculty of Applied Physics, University of Twente, P.O. Box 217, 7500 AE Enschede, The Netherlands

Received 28 July 1994; accepted for publication 27 January 1995

\begin{abstract}
The morphology of single domain $\mathrm{Si}(001) 2 \times 1$ surfaces has been investigated with scanning tunneling microscopy (STM). Using the model of Villain, Grempel and Lapujoulade, which is based on the microscopic properties of the surface, this vicinal surface is found to be thermally rough. On macroscopic scale, however, we did not observe any indication for the expected characteristic logarithmic divergence of the surface height-height correlation function. Possible causes for this discrepancy are discussed briefly.

Keywords: Equilibrium thermodynamics and statistical mechanics; Scanning tunneling microscopy; Silicon; Surface roughening; Surface structure; Vicinal single crystal surfaces
\end{abstract}

\section{Introduction}

Surfaces may undergo a roughening transition from a faceted to a rough surface above a certain temperature, as originally suggested by Burton, Cabrera and Frank in a classical paper [1]. This roughening transition of a crystal surface is characterized macroscopically by the disappearance of a facet of a given orientation from the equilibrium crystal shape. Microscopically the transition is characterized by the free energy of a step on the particular facet becoming zero, or alternatively by the appearance of strong fluctuations in the position of the facet.

Statistical mechanical theories classify solid surfaces as faceted or as rough depending on the behav-

\footnotetext{
${ }^{*}$ Corresponding author. Fax: +3153342657 .
}

ior of the fluctuations of the surface height. A convenient way to characterize the height fluctuations of the surface is the height-height correlation function between two points separated by a distance $r$ on the surface,

$G(r)=\left\langle[h(r)-h(0)]^{2}\right\rangle$,

where $r$ is the distance measured parallel to the substrate and $h(r)$ is the deviation measured perpendicular to the surface with respect to a reference plane. For rough surfaces at a temperature above or at the roughening temperature, $T_{\mathrm{I}}$, theory $[2,3]$ predicts that the height-height correlation function diverges logarithmically.

$G(r) \sim \ln (r), \quad T \geqslant T_{\mathrm{R}}$.

For facets, however, the surface height fluctuations are always finite.

$G(r) \sim$ const, $\quad T<T_{\mathrm{R}}$. 
For characterization of surface roughness by height-height correlation functions, diffraction techniques have been most commonly used until now $[4,5]$. From these techniques the height-height correlation function is obtained by detailed analysis of the diffraction peaks. The STM provides an alternative and more straightforward way to determine the height-height correlation function by calculating it directly from the measured surface height profiles [6].

In this study STM images of the single domain $\mathrm{Si}(001)$ surface are analyzed to test the predictions of statistical mechanics for rough surfaces in thermal equilibrium. On the other hand an alternative method, originally proposed by $\mathrm{Li}, \mathrm{Bartelt}$ and Williams [7] based on the microscopic properties of the surface, i.e. the kink creation energy and the step-step interaction energy, is used in order to determine whether the surface is thermally rough.

\section{Experimental}

The $\mathrm{Si}(001)$ samples we have used are $4^{\circ}$ misoriented in the [001] direction towards the [110] direction, giving rise to a nearly single domain surface consisting of terraces with lengths of about $40 \AA$. Before loading the samples in the ultra-high vacuum (UHV) chamber with a base pressure below $1 \times$ $10^{-10}$ Torr they were ultrasonically rinsed in ethanol. Prior to the cleaning of the Si sample, the sample and holder were outgassed at $800-900 \mathrm{~K}$ for several hours. The Si samples were cleaned inside the UHV chamber by repeated cycles of ion bombardment (Ar ions, $800 \mathrm{eV}$ ) and annealing at $1100 \mathrm{~K}$ for typically half an hour. This cleaning procedure is known to provide atomically clean $\mathrm{Si}(001) 2 \times 1$ surfaces [8].

The Si(001) surface reconstructs to form rows of dimerized atoms giving a $(2 \times 1)$ reconstruction. A slight misorientation (smaller than $2^{\circ}-3^{\circ}$ ) with respect to the [001] in the $[110]$ or $[-110]$ direction results in a vicinal surface with two types of monatomic step edges [9]. For very small $\left(\theta<0.1^{\circ}\right)$ miscut angles wavy (sinusoidal) step edges coexist with straight steps $[10,11]$. However, for larger miscut angle $\left(\theta>2^{\circ}-3^{\circ}\right)$ the $\mathrm{Si}(001)$ surface contains mainly biatomic steps $[12,13]$, resulting in a single domain $\mathrm{Si}(001)$ surface.
STM images have been taken on many places randomly chosen on the surface, covering areas ranging from 300 by $300 \AA^{2}$ to 5000 by $5000 \AA^{2}$. The images were taken with a sample bias of -2 to $-1.5 \mathrm{~V}$ and a tunneling current of $1 \mathrm{nA}$.

\section{Results and discussion}

Vicinal surfaces, that are surfaces misoriented by a small angle away from a low-index orientation, will be rough at a significantly lower temperature as compared to the roughening of the neighboring lowindex surface. The entropically favorable wandering of steps on the surface gives rise to this roughness. It is well known that an isolated step is not stable and roughens at any nonzero temperature. The relative stability of vicinal surfaces can be explained by repulsive interactions between the steps, which constrain the step wandering. The roughening temperature of vicinal surfaces is expected to increase with increasing miscut angle, i.e. with smaller terraces and stronger repulsive step-step interactions [5]. Whether a vicinal surface is rough or not at a certain temperature depends on the strength of the step-step interactions. Wandering steps give broad terrace length distributions corresponding to weak step-step interactions and rough surfaces. Smooth steps with narrow terrace length distributions correspond to strong interactions and hence the formation of facets. Whether the surface is thermally rough or not can be determined from the width of the terrace length distribution [7]. The roughening temperature, $T_{\mathrm{r}}$, of a stepped surface can be obtained from the following equation [2]

$$
\frac{\varepsilon_{\mathrm{s}}}{k_{\mathrm{B}} T_{\mathrm{r}}} \exp \left(\varepsilon / k_{\mathrm{B}} T_{\mathrm{r}}\right)=\frac{\pi^{2}}{2},
$$

where $\varepsilon$ is the kink formation energy of the step and $\varepsilon_{\mathrm{s}}$ is the energetic step-step interaction per lattice spacing displacement, i.e. the energy cost per unit length to move a step by one unit cell.

In the case of single domain vicinal $\mathrm{Si}(001)$ surfaces, we assume the interactions between the steps to be repulsive of the general form $U(L)=c L^{-2}$ $[14,15]$, where $L$ is the separation between two steps. Step-step interactions of this particular form give 
rise to a Gaussian terrace length distribution, in good agreement with our observations. (Note that the repulsive step-step interactions on double domain Si(001) surfaces display a $\ln (L)$ dependence due to the anisotropic stress tensor [16].) The energy $\varepsilon_{\mathrm{s}}$ is then given by

$\varepsilon_{\mathrm{s}}=c\left(\frac{1}{(L-1)^{2}}+\frac{1}{(L+1)^{2}}-\frac{2}{L^{2}}\right) \approx \frac{6 c}{L^{4}}$.

In the case of repulsive interactions of the form $L^{-2}$ the terrace length distribution is a Gaussian with a width $\sigma$ [17],

$\sigma=\left(\frac{k_{\mathrm{B}} T\left\langle k^{2}\right\rangle}{48 c}\right)^{1 / 4} L$,

The value $\left\langle k^{2}\right\rangle$ is the local mean square length of a kink or the diffusivity of a kink and is defined as [18]

$\left\langle k^{2}\right\rangle=\frac{2 \exp \left(-\varepsilon / k_{\mathrm{B}} T\right)}{\left[1-\exp \left(-\varepsilon / k_{\mathrm{B}} T\right)\right]^{2}}$.

By combining Eqs. (4), (5) and Eq. (7) and substitution of the resulting equation into Eq. (6) gives a critical Gaussian width of the terrace length distribution at the roughening transition

$\sigma_{\mathrm{R}} \approx\left(\frac{1}{2 \pi^{2}}\right)^{1 / 4} \approx 0.47 \mathrm{au}$.

When the Gaussian width of the terrace length distribution exceeds $\sigma_{R}$, the surface will be rough, whereas for widths smaller than $\sigma_{\mathrm{R}}$ the surface will be a facet.

Several STM images of various sizes of a nearly single domain $\mathrm{Si}(001)$ surface are shown in Figs. 1a-1c. The terrace length distribution determined from these STM images is shown in Fig. 2a. The distribution can be fitted well with a Gaussian width of $\sigma \sim 1.8$ au (see the solid line in Fig. 2a, 1 $\mathrm{au}=3.84 \AA$ ). This value is much larger than the critical width $\sigma_{\mathrm{R}}$, so the $4^{\circ}$ misoriented $\mathrm{Si}(001)$ surfaces observed in room temperature STM images must be thermally rough. We also know from high temperature STM images that the freeze in temperature $T_{\mathrm{f}}$ of vicinal $\mathrm{Si}(001)$ surfaces lies in the range $600-700 \mathrm{~K}$ [19]. From this we conclude that the
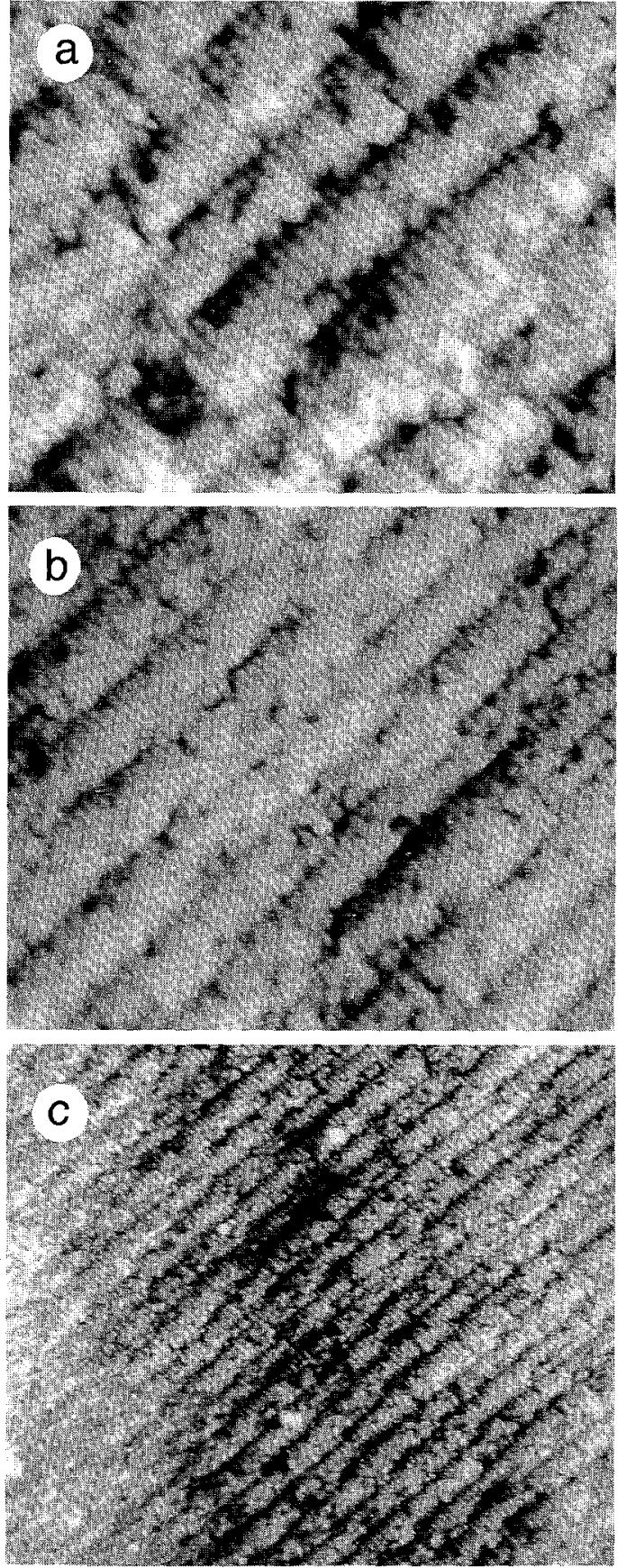

Fig. 1. Scanning tunneling microscopy image of a single domain $4^{\circ}$ misoriented $\mathrm{Si}(001)$ surface. Tunneling current was $1 \cdot \mathrm{nA}$ and the bias voltage -2 V. (a) Scan size $320 \times 350 \AA^{2}$. (b) Scan size $470 \times 570 \AA^{2}$. (c) Scan size $600 \times 700 \AA^{2}$. 

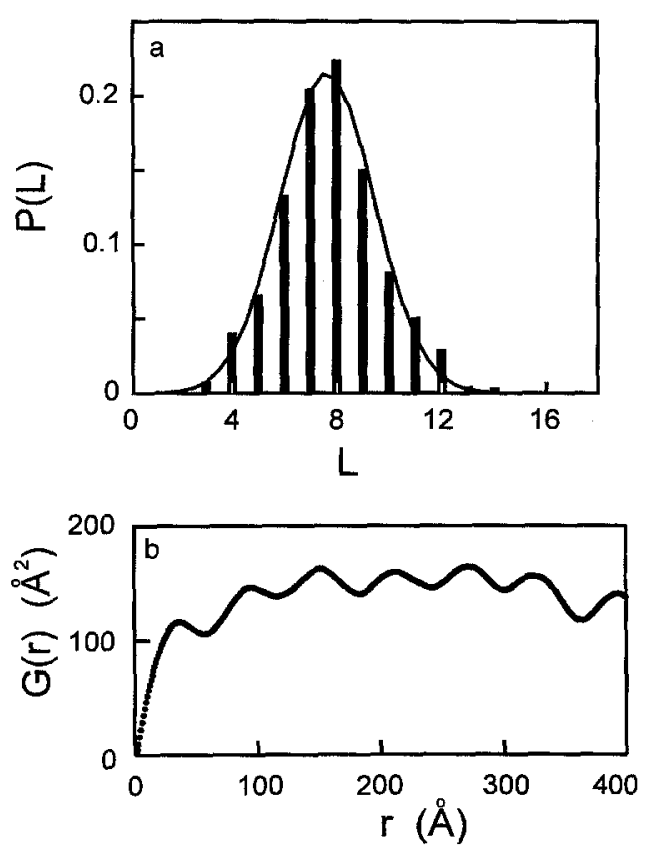

Fig. 2. (a) Normalized terrace length distribution of $4^{\circ}$ misoriented $\mathrm{Si}(001)$ surface. (b) Height-height correlation function of $4^{\circ}$ misoriented $\mathrm{Si}(001)$ surface.

roughening temperature of $4^{\circ}$ misoriented $\mathrm{Si}(001)$ surfaces must lie below $600 \mathrm{~K}$.

Because the surface is thermally rough, a logarithmic divergence of the height-height correlation function is expected, see Eq. (2). From many STM images (see e.g. Figs. 1a-1c) the height-height correlation function, see Eq. (1), is calculated. The tilt of the images is removed by fitting a plane through the data using the least-squares method and subtracting this plane from the data. The height-height correlation function is calculated only in the fast scan direction for $r$ values smaller than half of the scan size. A plot of the height-height correlation function calculated from STM images as shown in Fig. 1 is depicted in Fig. 2b. The height-height correlation first increases rapidly and then starts to oscillate. The appearance of the oscillations can simply be understood as brought about by the typical length of the terraces. However, the predicted logarithmic divergence of the height-height correlation function is not observed on this length scale. The height-height correlation tends to flatten out.

Obviously the behavior of the height-height cor- relation function of a real surface is not in agreement with the logarithmic divergence expected from the statistical mechanics for rough surfaces in thermal equilibrium. Several reasons can be suggested to explain this discrepancy. The length scale we are looking at can be too small to observe the crossover to logarithmic behavior of the height-height correlation function. To check the behavior on larger length scales we have analyzed low energy electron microscopy (LEEM) images of Si(001) surfaces with sizes up to $8 \mu \mathrm{m}[10,11]$. However, on this scale the height-height correlation functions do not show the logarithmic divergence either. The preparation of the sample is also a point to consider. The surface may not have been fully equilibrated during annealing. The length scale on which the surface is in equilibrium grows with some (weak) power of time depending on the exact mechanism of mass transfer [20-26]. An other reason for not observing the logarithmic divergence can be the unavoidable presence of defects on the surface. These defects can play an important role by acting as pinning centra and can disturb the behavior of the surface height fluctuations.

\section{Conclusions}

We have investigated the morphology of single domain $\mathrm{Si}(001)$ surfaces with scanning tunneling microscopy. Using the model of Villain, Grempel and Lapujoulade, which is based on the microscopic properties of the surface, we find that this vicinal surface is thermally rough. However, in contradiction with theory the surface height-height correlation does not show the expected logarithmic divergence on length scales up to a few microns.

\section{References}

[1] W.K. Burton, N. Cabrera and F.C. Frank, Philos. Trans. R. Soc. (London) A 234 (1951) 299.

[2] J. Villain, D.R. Grempel and J. Lapujoulade, J. Phys. F 15 (1985) 809.

[3] J.D. Weeks, in: Ordering in Strongly Fluctuating Condensed Matter Systems, Ed. T. Riste (Plenum, New York, 1980) p. 293.

[4] E.H. Conrad and T. Engel, Surf. Sci. 299/300 (1994) 391.

[5] J. Lapujoulade, Surf. Sci. Rep. 20 (1994) 191. 
[6] J.L. Goldberg, X.-S. Wang, N.C. Bartelt and E.D. Williams, Surf. Sci. 249 (1991) L285.

[7] Bin Li, N.C. Bartelt and E.D. Williams, preprint.

[8] H.J.W. Zandvliet, H.B. Elswijk, E.J. van Loenen and I.S.T. Tsong, Phys. Rev. B 46 (1992) 7581.

[9] D.J. Chadi, Phys. Rev. Lett. 59 (1987) 1691.

[10] R.M. Tromp and M.C. Reuter, Phys. Rev. Lett. 68 (1992) 820.

[11] R.M. Tromp and M.C. Reuter, Phys. Rev. B 47 (1993) 7598.

[12] B.S. Swartzentruber, N. Kitamura, M.G. Lagally and M.B. Webb, Phys. Rev. B 47 (1993) 20.

[13] P.E. Wierenga, J.A. Kubby and J.E. Griffith, Phys. Rev. Lett. 59 (1987) 2168.

[14] N.C. Bartelt, T.L. Einstein and E.D. Williams, Surf. Sci. 240 (1991) L591.

[15] E.D. Williams and N.C. Bartelt, in: Proc. workshop on Surface Disordering, Eds. R. Jullien, J. Kertesz, P. Meakin and D.E. Wolf (Nova Science, 1992) p. 103.

[16] O.L. Alerhand, D. Vanderbilt, R.D. Meade and J.D. Joannopoulos, Phys. Rev. Lett. 61 (1988) 1973.
[17] $\sigma$, often referred to as the width, is the standard deviation of the Gaussian, $P(x)=(1 / \sigma \sqrt{(2 \pi)}) \exp \left(-x^{2} / 2 \sigma^{2}\right)$.

[18] N.C. Bartelt, T.L. Einstein and E.D. Williams, Surf. Sci. 276 (1992) 308

[19] H.J.W. Zandvliet, H. Wormeester, D.J. Wentink, A. van Silfhout and H.B. Elswijk, Phys. Rev. Lett. 70 (1993) 2122.

[20] N.C. Bartelt, J.L. Goldberg, T.L. Einstein and E.D. Williams, Surf. Sci. 273 (1992) 252.

[21] W.W. Mullins, J. Appl. Phys. 28 (1957) 333.

[22] W.W. Mullins, J. Appl. Phys. 30 (1959) 77.

[23] W.W. Mullins, in: Metal Surfaces: Structure, Energetics and Kinetics (Am. Soc. Metals, Metal Park, OH, 1963) p. 17.

[24] G.S. Bales and A. Zangwill, Phys. Rev. B 41 (1990) 5500.

[25] A. Pimpinelli, J. Villain, D.E. Wolf, J.J. Metois, J.C. Heyraud, I. Elkinani and G. Uimin, Surf. Sci. 295 (1993) 143.

[26] N.C. Bartelt, R.M. Tromp and E.D. Williams, Phys. Rev. Lett. 73 (1994) 1656. 